\title{
The hippocampal fimbria of cuprizone-treated animals as a structure for studying neuroprotection in multiple sclerosis
}

\author{
M. Kipp • A. Norkus $\cdot$ B. Krauspe $\cdot$ T. Clarner $\cdot$ \\ K. Berger $\cdot$ P. van der Valk $\cdot$ S. Amor $\cdot$ C. Beyer
}

Received: 5 January 2011/Revised: 17 February 2011/Accepted: 11 April 2011/Published online: 24 April 2011

(C) The Author(s) 2011. This article is published with open access at Springerlink.com

\begin{abstract}
Objective and design It has been demonstrated that changes in the normal-appearing white matter (NAWM) in multiple sclerosis precede the appearance of classical lesions. The understanding of NAWM biology in an established disease model might help to clarify why some of them progress to active demyelinating lesions.

Material or subjects C57BL6 male mice (19-21 g) were used in this study.

Treatment Demyelination was induced by feeding mice a diet containing $0.2 \%$ cuprizone for up to 5 weeks.

Methods Routine stainings (luxol fast blue, and hematoxylin and eosin) and immunohistochemistry were performed to assess myelin status and the inflammatory infiltrate.

Results We demonstrated that, in the toxic demyelination cuprizone model, the corpus callosum is severely demyelinated after a 5-week cuprizone challenge (acute
\end{abstract}

Responsible Editor: Graham Wallace.

M. Kipp and A. Norkus contributed equally to this work.

M. Kipp ( $\square)$ · A. Norkus · B. Krauspe $\cdot$ T. Clarner

K. Berger - C. Beyer

Institute of Neuroanatomy, Faculty of Medicine, RWTH Aachen

University, Wendlingweg 2, 52074 Aachen, Germany

e-mail: mkipp@ukaachen.de

M. Kipp · P. van der Valk $\cdot$ S. Amor

Department of Pathology, VU University Medical Center,

Amsterdam, The Netherlands

S. Amor

Neuroimmunology Unit, Neuroscience Centre,

Blizard Institute of Cell and Molecular Science,

Barts and The London School of Medicine and Dentistry,

Queen Mary University of London, London, UK demyelination) whereas the fimbria of the hippocampus appear normal in routine myelin stainings. Microgliosis but not astrogliosis is evident after acute demyelination in the fimbria. Interestingly, both regions, the fimbria and the corpus callosum, demonstrated early oligodendrocyte apoptosis as well as intense microglia accumulation and activation. However, only the corpus callosum progresses to actively demyelination lesions whereas the fimbria does not.

Conclusions The applied model appears suitable for elucidating pathways which promote progression of affected tissue to an active lesion.

Keywords CNS inflammation · Inflammatory models

\section{Introduction}

Multiple sclerosis (MS) is the most common neurological disorder in young adults in the Western world. In addition to the well-characterized inflammatory white matter, histopathological and imaging studies have suggested that the so-called 'normal-appearing white matter' (NAWM) has significant abnormalities [1]. Axonal injury, blood-brain barrier changes and reactive astro- and microgliosis have been reported at the histological level [2-4]. Little is known about the molecular changes taking place in the NAWM in MS. It has recently be suggested that NAWM in MS is in a subtle balance between inflammation and neuroprotection [5]. Therefore, one might expect that the understanding of molecular changes within the NAWM could be exploited to develop new treatments.

In this study, we used the well-characterized toxic cuprizone model. Cuprizone ingestion in mice induces a highly reproducible demyelination of distinct brain regions, 
such as the corpus callosum (CC) [6]. Some regions, however, appear to be resistant to the toxin challenge, e.g. the fimbria of the hippocampus [7]. The aim of the study was (1) to define whether the fimbria fulfils histological criteria of the NAWM in an established disease model and (2) to get a better understanding of 'NAWM-lesion' development in this particular animal model.

\section{Materials and methods}

Research and animal care procedures were approved by the Review Board for the Care of Animal Subjects of the district government (North Rhine-Westphalia, Germany). Demyelination was induced by feeding 8 -week-old male mice (C57BL/6; 19-21 g) a diet containing $0.2 \%$ cuprizone (bis-cyclohexanone oxaldihydrazone; Sigma-Aldrich Inc., USA) mixed into a ground standard rodent chow for the indicated periods. Tissue preparation and immunohistochemistry (IHC) were performed as published in detail previously [7, 8]. Anti-proteolipid protein (PLP) antibodies (mouse IgG, 1:500; Serotec, Germany) were used to detect myelin, anti-Iba1 antibodies (rabbit IgG, 1:250; Wako, Germany) to detect microglia/macrophages, and anti-glial fibrillary acidic protein (GFAP) antibodies (rabbit IgG, 1:1,000; EnCor, USA) were used to visualize astrocytes. Anti-olig2 antibodies (Millipore, Germany) were used to detect oligodendrocytes.

\section{Results}

In a first set of experiments, animals $(n=5$ per experimental group) were fed cuprizone for 5 weeks to induce acute demyelination of the CC. Definite loss of PLP immunoreactivity could be observed in the CC (Fig. 1b/e), whereas other regions appeared unaffected, such as the fimbria of the hippocampus (Fig. 1c/f). Both regions included in the subsequent study are indicated by the arrow and star in Fig. 1d. Quantitative measurement of PLP staining intensity confirmed our observational results. There was a significant decrease in PLP staining intensity in the CC (control $69.2 \pm 6.8$ [mean \pm SEM] vs. 5 weeks cuprizone $19.4 \pm 4.4 ; P<0.005)$. This was not observed in the fimbria (control $65.3 \pm 5.1$ vs. 5 weeks cuprizone 61.0.4 $\pm 3.2 ; P=0.49$ ). Luxol fast blue staining showed similar results (data not shown). To further investigate both regions on the cellular level, we visualized microglia by anti-Ibal and (activated) astrocytes by anti-GFAP antibodies, respectively. The quantification of fimbrial Iba $1^{+}$ and $\mathrm{GFAP}^{+}$cell numbers after acute cuprizone-induced demyelination is presented in Fig. 1u. Microglia were uniformly distributed in the $\mathrm{CC}$ (Fig. 1g) and fimbria
(Fig. 1i) of control animals while a sustained increase in $\mathrm{Iba}^{+}$cell numbers was present in the demyelinated CC (Fig. 1h) and, although to a lesser extend, in the fimbria (Fig. 1j). After 5 weeks cuprizone, higher numbers of $\mathrm{GFAP}^{+}$astrocytes compared to controls were seen within the CC (Fig. 1k/l) but not in the fimbria (Fig. $1 \mathrm{~m} / \mathrm{n}$ ).

From our observation that definite microgliosis can be observed within the fimbrial region after acute cuprizoneinduced demyelination, we assumed that the fimbria is affected like the CC by the toxin per se, but does not develop to a 'classically' demyelinated lesion. To test for this hypothesis, animals ( $n=6$ per experimental group) were fed cuprizone in a second experiment up to 5 weeks and sacrificed on day 2, week 1, week 2 and week 5 after the start of the cuprizone diet. Cells with morphological characteristics typical for apoptosis such as condensed and/ or fragmented nuclei were seen at week 1 in both regions included in the study (Fig. 1o/p). Control animals fed with powdered food without cuprizone were lacking apoptotic cells (data not shown). In a previous study, immunohistochemistry for detection of CNPase confirmed that these apoptotic cells are oligodendrocytes [9]. Anti-Iba-1 IHC revealed that pronounced microgliosis is evident in both regions after week 1 . In control animals, Iba $1^{+}$cells displayed a typical ramified morphology indicating a resting state (Fig. 1q). At week 1 (Fig. 1s) and week 2 (Fig. 1t), processes of microglia cells within the fimbria appeared to be retracted and cell bodies were swollen, which is frequently associated with microglia activation [10]. Comparable morphological changes of microglia were evident in the $\mathrm{CC}$ at weeks 1 and 2 . While the magnitude of microgliosis progressed in the CC until week 5 (Fig. 1h), a reduction of microglia cell numbers could be observed in the fimbria. Only a moderate activation of fimbrial astrocytes was detectable at week 2 (increased number and swollen processes), whereas at all other time points in the study, astrocytic cell parameters were the same as observed in the fimbria of control animals. Within the CC, astrocytosis started at week 2; however, in contrast to the fimbria, it progressed in the CC until week 5. At week 2, the number of olig2-positive cells was dramatically decreased in the CC whereas no difference in oligodendrocyte cell numbers (and PLP staining intensity) was evident in the fimbria compared to control animals.

\section{Discussion}

Activation of microglia is a well-known characteristic histological feature of the NAWM in MS $[2,11]$. Whether activated microglia are beneficial or detrimental for disease progression is controversial $[11,12]$. In this study, we have shown that the fimbria of the hippocampus, which shows 


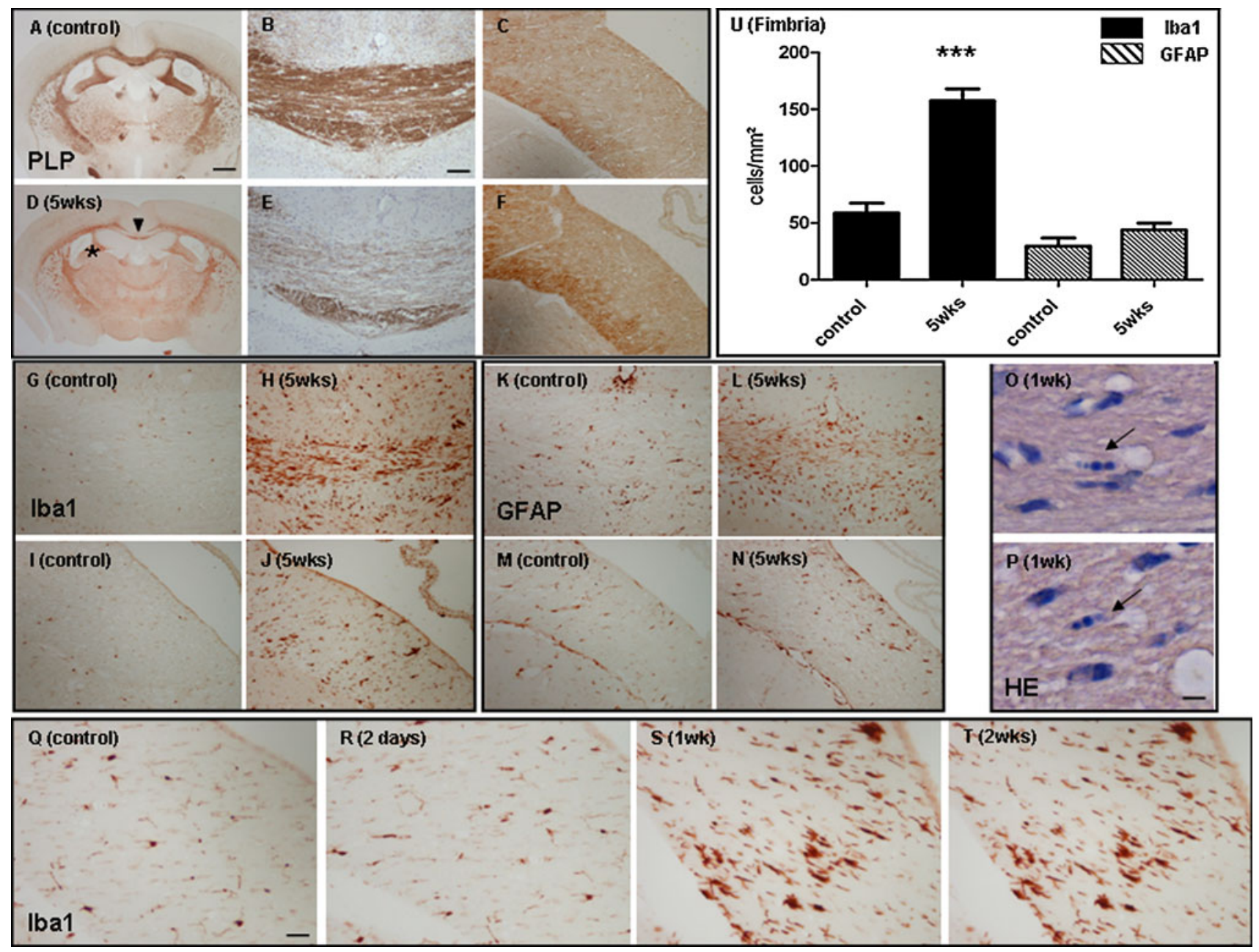

Fig. 1 Myelination and inflammatory cell composition in the medial corpus callosum (CC) and fimbrial region of the hippocampus. AntiPLP antibodies were used to visualize intact myelin in sections of control and cuprizone-treated animals (a-f). Anti-Iba1 and anti-GFAP antibodies were used to visualize microglia or astrocytes, respectively. Arrowhead in (d) indicates the medial part of the CC, which is shown in $(\mathbf{b} / \mathbf{e}, \mathbf{g} / \mathbf{h}, \mathbf{k} / \mathbf{l}$ and $\mathbf{o})$ in higher magnification. The star in (d) indicates the fimbrial region of the hippocampus, which is shown

characteristic features of NAWM after acute cuprizoneinduced demyelination, is not protected from the toxic compound per se. Both regions, the fimbria and the $\mathrm{CC}$, [13] demonstrated early oligodendrocyte apoptosis and intense microglia accumulation and activation. However, only in the CC did we observe lesions that progressed to actively demyelination lesions, whereas such a course was not seen in the fimbria. Remyelination appears not to be the underlying mechanism of preserved myelination in the fimbria, since at all time points in the study anti-PLP staining intensity remained unchanged in this region.

It might be feasible that regional differences exist in microglia function, as previously suggested [14]. We assume that some intrinsic signaling contributes to a 'switch' from an anti-inflammatory to a pro-inflammatory in $(\mathbf{c} / \mathbf{f}, \mathbf{i} / \mathbf{j}, \mathbf{m} / \mathbf{n}, \mathbf{p}$ and $\mathbf{q - t})$ in higher magnification. Pictures $(\mathbf{o} / \mathbf{p})$ show hematoxylin-and-eosin-stained sections, illustrating apoptotic oligodendrocytes (arrows) within the CC and fimbria. Quantification of $\mathrm{Iba}^{+}$microglia and $\mathrm{GFAP}^{+}$astrocytes (fimbria) in control and 5 weeks' cuprizone-treated animals is given in $(\mathbf{u})$. $* * * P<0.001 ;$ scale bars: $500 \mu \mathrm{m}(\mathbf{a} / \mathbf{d}) ; 50 \mu \mathrm{m}(\mathbf{b} / \mathbf{c}, \mathbf{e} / \mathbf{f}, \mathbf{g}-\mathbf{n})$; $10 \mu \mathrm{m}(\mathbf{o} / \mathbf{p}) ; 25 \mu \mathrm{m}(\mathbf{q}-\mathbf{t})$

phenotype of microglia within the affected CC. The former phenotype is also called 'M1 or classically activated', the latter one 'M2 or alternatively activated' $[13,15]$. Therapeutic bolstering of this apparent regulation could therefore still be effective in halting the formation of new destructive lesions in human MS. In this situation, prevention of further damage rather than repair of existing damage could still be achieved.

\section{Summary}

The applied animal model with an existing regional diversity in the course of disease appears to be suitable for elucidating pathways which promote progression of the 
NAWM to an active lesion. We have focused on the fimbrial region of the hippocampus, since this brain region can readily be dissected without the need of costly equipment (such as laser-capture microscopy) for subsequent analyses on the molecular level.

Open Access This article is distributed under the terms of the Creative Commons Attribution Noncommercial License which permits any noncommercial use, distribution, and reproduction in any medium, provided the original author(s) and source are credited.

\section{References}

1. Filippi $\mathrm{M}$, et al. A magnetization transfer imaging study of normal-appearing white matter in multiple sclerosis. Neurology. 1995;45(3 Pt 1):478-82.

2. Allen IV, McKeown SR. A histological histochemical and biochemical study of the macroscopically normal white matter in multiple sclerosis. J Neurol Sci. 1979;41(1):81-91.

3. Fu L, et al. Imaging axonal damage of normal-appearing white matter in multiple sclerosis. Brain. 1998;121(Pt 1):103-13.

4. Silver NC, et al. Quantitative contrast-enhanced magnetic resonance imaging to evaluate blood-brain barrier integrity in multiple sclerosis: a preliminary study. Mult Scler. 2001;7(2):75-82.

5. Zeis T, et al. Normal-appearing white matter in multiple sclerosis is in a subtle balance between inflammation and neuroprotection. Brain. 2008;131(Pt 1):288-303.
6. Kipp M, et al. The cuprizone animal model: new insights into an old story. Acta Neuropathol. 2009;118(6):723-36.

7. Norkute A, et al. Cuprizone treatment induces demyelination and astrocytosis in the mouse hippocampus. J Neurosci Res. 2009;87(6):1343-55.

8. Pott $\mathrm{F}$, et al. Cuprizone effect on myelination, astrogliosis and microglia attraction in the mouse basal ganglia. Brain Res. 2009;1305:137-49.

9. Hesse A, Wagner M, Held J, Brück W, Salinas-Riester G, Hao Z, Waisman A, Kuhlmann T (2010) In toxic demyelination oligodendroglial cell death occurs early and is FAS independent. Neurobiol Dis. 37(2): 362-369.

10. Heppner FL, et al. Vitamin $\mathrm{E}$ induces ramification and downregulation of adhesion molecules in cultured microglial cells. Glia. 1998;22(2):180-8.

11. Howell OW, Rundle JL, Garg A, Komada M, Brophy PJ, Reynolds R (2010) Activated microglia mediate axoglial disruption that contributes to axonal injury in multiple sclerosis. J Neuropathol Exp Neurol. 69(10): 1017-1033.

12. Napoli I, Neumann H (2010) Protective effects of microglia in multiple sclerosis. Exp Neurol. 225(1): 24-28.

13. Gordon S. Alternative activation of macrophages. Nat Rev Immunol. 2003;3(1):23-35.

14. de Haas AH, Boddeke HW, Biber K. Region-specific expression of immunoregulatory proteins on microglia in the healthy CNS. Glia. 2008;56(8):888-94.

15. Rivest $\mathrm{S}$. Regulation of innate immune responses in the brain. Nat Rev Immunol. 2009;9(6):429-39. 